\title{
The benefit and challenges of non-spherical refinements - NoSpherA2
}

\author{
F. Kleemiss ${ }^{1}$, S. Grabowsky², O. V. Dolomanov ${ }^{3}$, H. Puschmann ${ }^{3}$, M. Bodensteiner ${ }^{1}$ \\ ${ }^{1}$ University of Regensburg, Department for Chemistry and Pharmacy, Universitätsstraße 31, 93053 Regensburg, Germany, \\ ${ }^{2}$ University of Bern, Department of Chemistry, Biochemistry and Pharmacy, Freiestrasse 3, 3012 Bern, Switzerland, ${ }^{3}$ OlexSys Ltd., \\ Chemistry Department, Durham University, DH1 3LE, United Kingdom
}

florian.kleemiss@ur.de

The development of new procedures to refine experimental diffraction data lead to an increased number of individual software packages to perform these analyses. They may require setup of specific input files, learning of configuration files and sometimes result in file-types unique to each package, which can make comparisons, changes between methods and the overall workflow time consuming and only available to a trained specialist. NoSpherA2 - Non-Spherical-Atoms-in-Olex2 [1] - provides a possibility to interface any type and source of atomic form factors to the refinement engine olex2.refine [2], itself based on the cctbx [3].

This interface makes it possible to combine any level of sophistication in the calculation of the form factors, ranging from tabulated spherical atoms to tailor-made form factors from quantum mechanical calculations with the established refinement engine. Restraints, constraints, disorder modelling, solvent masking and intuitive handling using the well-known Graphical-User-Interface of Olex2 [4] are main advantages, which in combination with easy selection of options, automatic completion of CIFs and no required manual handling of input files make the treatment of diffraction data using non-spherical models easier than ever.

While NoSpherA2 provides a variety of possibilities and generally better results of refinements (compare Figure 1), some questions about handling of various structures arise: Is it possible to mix-and-match different approaches? How to handle network compounds like MOFs and inorganic Salts? If we describe non-spherical density distribution of atoms, what information possibly left in the data might need improved treatment? What resolution is required to use NoSpherA2?

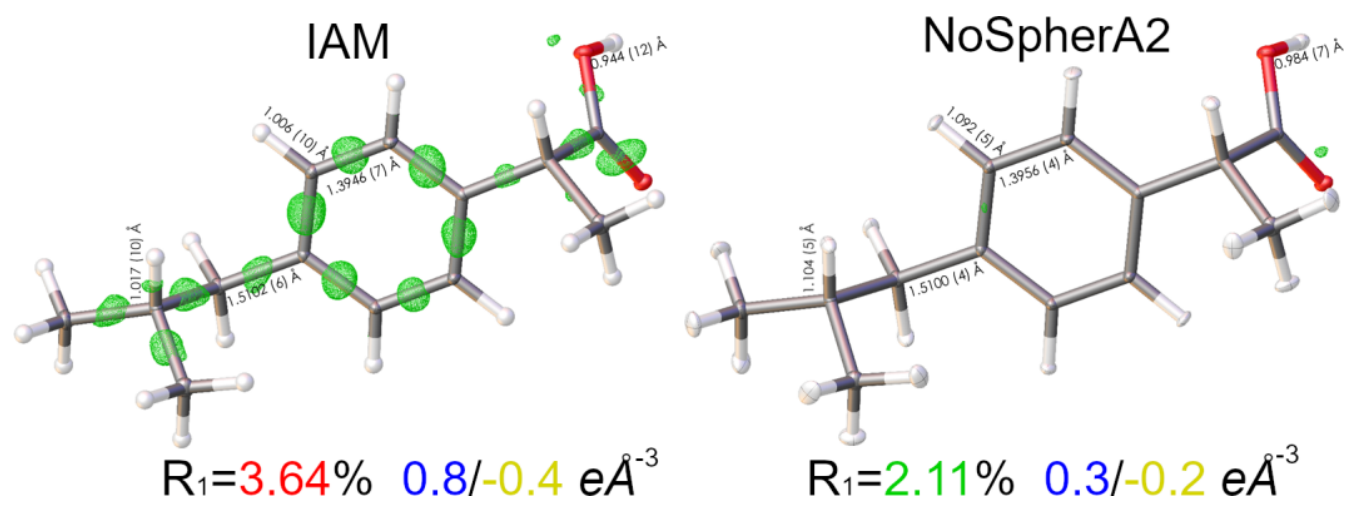

Figure 1. Comparison of Refinement results from Spherical (left) and Non-Spherical Model (right).

The benefits, common practice and strategies to tackle problems when using NoSpherA2 will be presented with examples and the philosophy of the development: Making the best-suited model available for the refinement task to obtain the best possible results without the need of individual file-conversion, in-depth training or specialized extra software.

[1] Kleemiss, F., Dolomanov, O. V., Bodensteiner, M., Peyerimhoff, N., Midgley, L., Bourhis, L. J., Genoni, A., Malaspina, L. A., Jayatilaka, D., Spencer, J. L., White, F., Grundkötter-Stock, B., Steinhauer, S., Lentz, D., Puschmann, H., Grabowsky, S. (2021), Chem. Sci. 12, $1675-1692$.

[2] Bourhis, L. J., Dolomanov, O. V., Gildea, R. J., Howard, J. A. K., Puschmann, H. (2015), Acta Cryst. A71, 59-75.

[3] Grosse-Kunstleve, R.W., Sauter, N.K., Moriarty, N. W., Adams, P. D. (2002), J. Appl. Cryst. 35, 126-136.

[4] Dolomanov, O. V., Bourhis, L. J., Gildea, R. J., Howard, J. A. K., Puschmann, H. (2009), J. Appl. Cryst. 42, $339-341$.

Keywords: Non-Spherical, Refinement, Software, HAR, Quantum Crystallography 